\title{
Report the Effective of Integrated Palliative Care using for Advanced Cancer as Second-Line or Later Treatment by Herbal Extractive Medicine
}

\author{
Xianyong Li ${ }^{1,2 *}$, Pu Rong ${ }^{1 *}$, Lingyan Zhang ${ }^{1}$, Weihua Yin ${ }^{1}$ and Cui Yu ${ }^{2}$ \\ ${ }^{1}$ Department of Oncology, Chengdu Fuxing Hospital, China \\ ${ }^{2}$ Department of Radiology, Chengdu Fuxing Hospital, China
}

*Corresponding author: Pu Rong, Department of Radiology, Chengdu Fuxing Hospital, China.

Received Date: April 09, 2019

Published Date: April 29, 2019

\section{Abstract}

Background: The on-going treatments of unresectable pancreatic, hepatocellular and late-stage lung cancer (PC \HCC \LC) are less effectiveness as second-line or later treatment. However, the palliaitve care is alternative option. Here, we reported the patients who received palliative care by herbal extractive medicines (CHM).

Methods: 13 pancreatic, 31 hepatocellular and 168 lung cancer patients were enrolled. CHM (Senghuang, SH; Xianhe Baijiang, XB), which approved by the China Food and Drug Administration (CFDA), hyperthermia and arginine were initiated. Survival time, quality of life (QOL) and toxicity were evaluated.

Result: The average ECOG score improved and no severe hematology and digestion side-effects were observed. In PC group, the median and the average survival time were 5.1 and 6.5 months; The 3-, 6-, and 10-month survival rates were $92.3 \%, 46.2 \%$, and $30.8 \%$, respectively. The longest survival time was 16.7 months and patient still alive. In HCC group, the average and median survival time were 12.48 and 5.03 months; The 3-, 6-, and 12 -month survival time rates were $77.42 \%, 38.71 \%$, and $29.03 \%$, respectively. The longest survival time was 84.2 months. In LC group, the 6 months, 1-, 2- and 5-year survival time rates were 33.93\%, 19.05\%, 14.29\% and 4.17\%, respectively. Meanwhile, in lung cancer group, once the survival time was over 6 months, the average and median survival time were 29.98 and 14.80 months; and the 1-, 2- and 5-year survival time rates were up to $56.14 \%, 42.11 \%$ and $12.28 \%$, respectively.

Conclusion: CHM can be considered as complementary and alternative medicine that provide moderate effective and low toxicity for advanced cancer.

Keywords: Palliative care; Herbal extracted medicine; Advanced cancer

\section{Introduction}

Lung cancer (LC), hepatocellular carcinoma (HCC) and pancreatic cancer (PC) are severe malignant cancer. According to GLOBOCAN 2012 report, accounting for $19.45 \%$ of all newly diagnosed pancreatic cancer cases in China and $19.27 \%$ of all deaths from pancreatic cancer worldwide [1]. Usually, surgery, chemotherapy, radiation therapy and target therapy are used as the first line treatment. Surgery should have been a potentially curative therapy for early stage lung cancer, hepatocellular carcinoma and pancreatic cancer [2], however, many patients are not eligible for surgery [3]. For instant, surgical resection may offer a 5-year survival up to $70 \%$ in liver cancer patients, but for patients with advanced disease, surgical resection is an option for less than
$20 \%$ [4]. Recently, PD-1 and PD-L1 anti-body therapy had been approved in lung and liver cancer, but no standard treatments for such stage patients after $1^{\text {st }} / 2^{\text {nd }}$ line failure are recommended. Nowadays, the median overall survival (OS) for late-stage liver, lung and pancreatic cancer were 7.9 months, 8.2 and 5.3 months, respectively [1,5-8]. Integrated palliative care is focused and seems as rational anti-cancer way for advanced cancer patients with low toxicity and moderate effectiveness. Herbal extractive medicines represent relatively low-toxicity and anti-cancer ability of multitargets by regulating tumor microenvironment and immune system [9-11]. According to clinical studies, while the late-stage patients who had been failure with second-line or later treatment, integrated palliative care that includes herbal extractive medicine 
was considered priority as a slavage treatment. Meanwhile, our previous laboratory studies have indicated that TGF- $\beta$, EGF, VEGF, IL-10 cytokines expression and mico-vessel density could be downgraded by Senghuang capsule ( $\mathrm{SH}$ ) and Xianhe Baijiang capsule (XB) that extracted from Ginseng, Herba Agrimonia, Hairyvein and Arginine etc in 4T1 and CT26 cell lines model.

In this study, herbal extractive medicines were given to advanced lung cancer, hepatocellular cancer and unresectable pancreatic cancer patients, and quailty of life (QOL), toxicity and survival time were observed.

\section{Methods}

\section{Patients}

In total, 212 late-stage cancer patients including 13 unresectable pancreatic cancer patients, 31 hepatocellular cancer patients and
168 lung cancer patients, who were failure with first line treatment or could not tolerate toxicity treatments, were enrolled. All patients had radiographic and pathological diagnosis. The retrospective study had been approved by Ethics Committee of Chengdu Fuxing Hospital.

\section{Drugs and treatment}

Herbal extractive medicines, which approved by the Chinese Food and Drug Administration (CFDA), hyperthermia and argine were used. Survival time, quality of life (ECOG score) and toxicity were observed. The prescription was shown in Figure 1: Arginine $15 \mathrm{ml}$, Senghuang capsule (SH) 1.80g and Xianhe Baijiang capsule (XB) $0.8 \mathrm{~g}$ Qid and hyperthermia $41-41.5^{\circ} \mathrm{C}$ Biw for four weeks as one cycle. Previous test by HPLC shows that the main ingredients of $\mathrm{SH}$ and XB consist of flavonoid, saponins and glucosides etc (Figure 1).

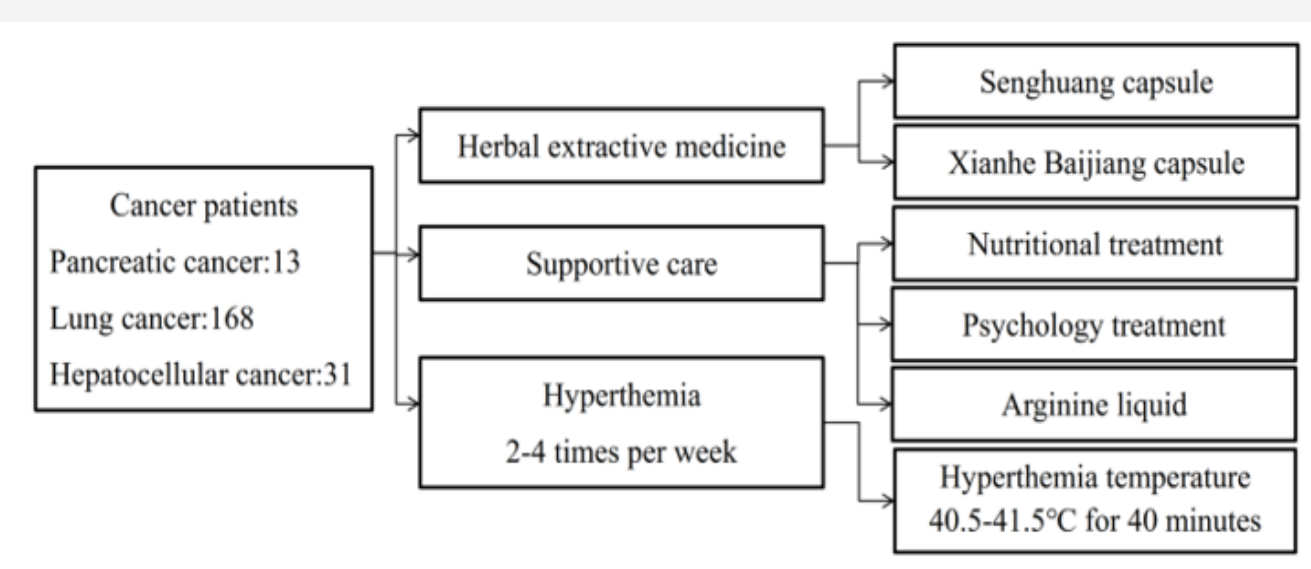

Figure 1: Herbal medicines, hyperthermia and supportive care were combined and used as complementary and alternative medicine. Herbal extractive medicine and hyperthermia acted on regulating tumor growth. Supportive care provided moderate nutrition such as arginine, glutamine and electrolyte, and psychology therapeutic intervention was given at the beginning.

\section{Data collection and safety \& efficacy assessment}

Prior therapies, patient's condition and drugs' dose, treatment information (i.e. current dose, dose adjustments), adverse events of LC, PC and HCC were recorded. ECOG score were documented at baseline and/or follow-up. Efficacy criteria included overall survival (OS) and quality of life (QOL). Adverse events were recorded and graded by National Cancer Institute Common Terminology Criteria version 3.0 (CTCAE 3.0).

\section{Result}

\section{Patient characteristics and treatment}

A total of 212 patients were enrolled including liver cancer (HCC), pancreatic cancer (PC) and lung cancer (LC) in late stage. Patients demographic and baseline characteristics was shown in Table 1. The median age was 58.0(PC), 62.7(LC) and 55.0(HCC) yearold, respectively, and the majority of patients were male $(53.8 \% \mathrm{PC}$, 64.9\% LC and 77.4\% HCC). 4.7\% (10/212) patients were tumornode-metastasis (TNM) stage at III, and 95.3\% (202/212) patients were stage IV. According to the Eastern Cooperative Oncology Group (ECOG) score evaluation criteria, 92.3\%(PC), 34.8\%(HCC) and $36.3 \%(\mathrm{LC})$ were up to 2 score, and $7.7 \%(\mathrm{PC}), 65.5 \%(\mathrm{HCC})$ and $63.7 \%(\mathrm{LC})$ were at 3-4 score, respectively. Treatment plan was shown in Table2 (Table 1,2).

Table 1: Description of patients' distribution. Patients who were suffered from III-IV stage cancer or the first/second line treatment failure were enrolled. (HCC: hepatocellular carcinoma; PC: pancreatic cancer; LC: Lung cancer).

\begin{tabular}{|c|c|c|c|}
\hline & $\begin{array}{c}\text { Unresectable PC } \\
(\mathbf{n = 1 3})\end{array}$ & $\begin{array}{c}\text { Stage III-IV LC } \\
(\mathbf{n = 1 6 8})\end{array}$ & $\begin{array}{c}\text { Stage IV HCC } \\
(\mathbf{n}=\mathbf{3 1})\end{array}$ \\
\hline Median age & 58 & 62.7 & 55 \\
\hline Male & 7 & 109 & 24 \\
\hline Female & 6 & 59 & 7 \\
\hline \multicolumn{4}{|c|}{ ECOG Performance Status } \\
\hline 2 & 12 & 61 & 8 \\
\hline 4-Mar & 1 & 107 & 23 \\
\hline \multicolumn{5}{|c|}{ Prior-Treatment } \\
\hline 0 & 8 & 27 & 5 \\
\hline 2-Jan & 4 & 141 & 26 \\
\hline TNM Stage & \multicolumn{2}{|c|}{} \\
\hline III & 0 & 10 & 0 \\
\hline IV & 13 & 158 & 31 \\
\hline
\end{tabular}


Table 2: Treatment plan was shown. Arginine liquid, Senghuang capsule $(\mathrm{SH})$ and Xianhe Baijiang capsule $(\mathrm{XB})$ were given four times per day by oral use.

\begin{tabular}{|c|c|c|}
\hline & Treatment & Times \\
\hline Arginine Liquid & $15.0 \mathrm{ml}$ & Qid \\
\hline Senghuang Capsule (SH) & $0.8 \mathrm{~g}$ & Qid \\
\hline Xianhe Baijiang Capsule (XB) & $1.2 \mathrm{~g}$ & Qid \\
\hline Hyperthermia & Biw & \\
\hline Supportive Care & If needed & \\
\hline
\end{tabular}

\section{Safety assessment}

Safety data were reported in Table 3. Dyspepsia and skin injure were the most common adverse events. Dyspepsia was drug-related adverse events that was experienced $15.1 \%(\mathrm{n}=32)$ patients under grade 2; and no patients suffered over grade 3 on hematology as drug-related adverse events. Meanwhile, $10.9 \%$ patients $(n=23)$ experienced skin injure events duo to hyperthermia treatment. No patients were excluded caused by severe side-effect (Table 3).

Table 3: Drug-related side-effects were shown (all grades), Data were presented as count (percentage).

\begin{tabular}{|c|c|c|}
\hline & Grade 1-2 & Grade 3-4 \\
\hline Dyspepsia & $15.1(32)$ & 0 \\
\hline Skin Injury & $10.9(23)$ & 0 \\
\hline Hematology & & 0 \\
\hline RBC & $34.9(74)$ & 0 \\
\hline WBC & $63.7(135)$ & 0 \\
\hline PLT & $12.7(27)$ & 0 \\
\hline Treatment Emergent Death & 0 & 0 \\
\hline
\end{tabular}

\section{Efficacy}

First, for168 lung cancer patients. The 6 months, 1-, 2- and 5-year survival time rates were $33.93 \%, 19.05 \%, 14.29 \%$ and $4.17 \%$ (Table 4), respectively. However, once the survival time was extended over 6 months (57/168), the average and median survival time were up to 29.98 and 14.80 months (Table 5); and the 1-, 2- and 5-year survival time rate were up to $56.14 \%, 42.11 \%$ and $12.28 \%$ (Table 4), respectively. The longest survival time reached to 134 months (Table 5). According to ECOG criteria, QOL in $59.6 \%$ (34/57) patients were improved (Figure 2). Meanwhile, according to the data, such sub-group patients, who respond to herbal extractive medicine at least one month, could get better benefit from herbal extractive medicine based palliative care, and the drugrelated effectiveness would maintain. Second, for unresectable pancreatic cancer patients. The 3-, 6-, and 10-month survival rates were up to $92.3 \%, 46.2 \%$ and $30.8 \%$, respectively (Table 4 ). The median and the average survival time were up to 5.1 and 6.5 months. The longest survival time was 16.7 months and patient still alive (Table 5). According to ECOG, QOL in 53.8 \% (7/12) patients were improved (Figure 2). Third, for liver cancer patients. The 6-, $12-, 24$ months and 5-year survival time rates were up to $45.16 \%$, $29.03 \%, 12.90 \%$ and $3.23 \%$, respectively (Table 4 ). The average and median survival time were 12.48 and 5.03 months, respectively, and the longest survival time was 84.17 months (Table 5). According to ECOG, QOL in $28.5 \%$ (4/14) patients were improved and QOL in another $28.5 \%$ (4/14) patients were stable (Tables 4,5) (Figure 2).

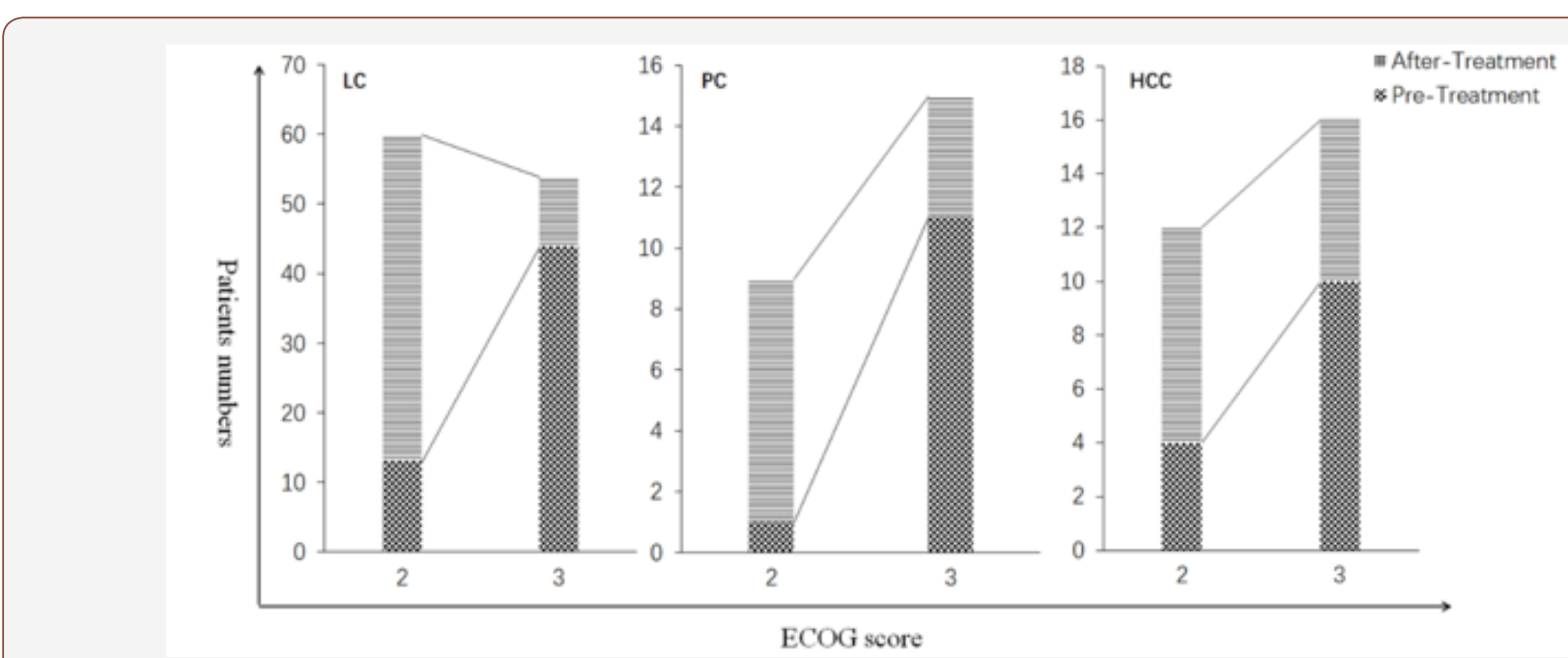

Figure 2: Comparison of ECOG score between pre-treatment and 6 months later for LC and HCC, or 3 months later for PC. According to the data, life quality of the patients has improved after treatment.

Table 4: Distribution of survival time of the LC, PC and HCC patients. The result shown that such terminal stage patients would reach acceptable survival time by using herbal extractive medicine as alternative treatment.

\begin{tabular}{|c|c|c|c|c|c|c|c|}
\hline & & $\mathbf{3}$ Months & $\mathbf{6}$ Months & $\mathbf{9}$ Months & $\mathbf{1 2}$ Months & $\mathbf{2 4}$ Months & $\mathbf{6 0}$ Months \\
\hline LC & All patients (\%) & & $33.93(57 / 168)$ & & $19.05(32 / 168)$ & $14.29(27 / 168)$ & $4.17(7 / 168)$ \\
\hline & $\begin{array}{c}\text { Once alive over } \\
6 \text { months (\%) }\end{array}$ & & & & $56.14(32 / 57)$ & $42.11(27 / 57)$ & $12.28(7 / 57)$ \\
\hline PC (\%) & & $92.3(12 / 13)$ & $46.2(6 / 13)$ & $30.8(4 / 13)$ & & & \\
\hline HCC (\%) & & & $45.16(14 / 31)$ & & $29.03(9 / 31)$ & $12.9(4 / 31)$ & $3.23(1 / 31)$ \\
\hline
\end{tabular}


Table 5: The median, average and longest survival time of Lung cancer patients, pancreatic cancer and HCC were shown. According to the data, for the LC, 57 patients who alive over 6 month were observed and the average survival time was up to 29.98 months; for 13 PC and HCC patients, the median survival time was up to 6.5 months and 12.48 months, which reached the preview studies.

\begin{tabular}{|c|c|c|c|}
\hline & LC(Month) & PC(Month) & HCC(Month) \\
\hline Median Survival Time & 14.8 & 5.1 & 5.03 \\
\hline Average Survival Time & 29.98 & 6.5 & 12.48 \\
\hline Longest Survival Time & 134 & 16.7 & 84.17 \\
\hline
\end{tabular}

\section{Discussion}

This paper reported the patients who fail to on-going anti-cancer treatments. Approximately 30\% of cancer patients who received first-line therapy need further subsequent treatment [12]. Tyrosine kinase inhibitor (TKI) such as Gefitinib, Solafinib or Laptinib have been shown to improve survival and symptoms in NSCLC and HCC. However, despite advances in pancreatic cancer therapy, the average survival rate at 5 years remains only $6 \%$ [13], especially for unresectable pancreatic cancer. Within these few treatment choices, adverse effects of recent drugs have further restricted patient clinical use [14], and their effectiveness for HCC and pancreatic cancer is not satisfactory. Nevertheless, herbal extractive medicines represent relatively low-toxicity and anti-cancer ability of multi-targets by regulating tumor microenvironment and immune system, which is preferred as second or later treatment and manage symptoms [15]. In clinical trials, it was proved by multidisciplinary teams, but the intensity of interventions varied considerably. Based on some clinical trials, integrated palliative care decreased overall mortality (HR $0.77 ; 95 \%$ CI 0.61 to 0.98 ), and short-term (1-3 months) effects of integrated palliative care on QOL was improved, but longer-term (6-12 months) effects on QOL was not improved [16-20].

Recently, once the definition of palliative care intervention characteristics was interpreted clearly, the more precise understanding of the impact of integrated palliative and oncology care on outcomes would approach [21]. Study by Temel et al, 151 NSCLC patients were delivered to early palliative care integrated with ongoing oncology care or to standard oncology care alone group in order to prove the importance of supportive care. Not only the patients who received early palliative care had better quality of life and less depression, but also were less likely to receive useless chemotherapy ( $33 \%$ vs. $54 \%$ in the standard chemo-treatment, $p=$ 0.05). The patients, who received early palliative care, lived longer compared with standard treatment group (11.6 months vs. 8.9 months, $\mathrm{P}=0.02$ ) [12,17]. Meanwhile, palliative care for pancreatic cancer were recommended in the course of illness and concurrently with active treatment $[22,23]$. The use of Chinese herbal medicines (CHM) in symptom management for cancer palliative care is common in Chinese populations and clinical evidence on their effectiveness is already to be confirmed, and quality of life among cancer patients are affected. More than that, herbal medicines acted on the tumor and micro-environment though multi-targets [11]. Depend on our laboratory research, active ingredients, which were extracted from Ginseng, Herba Agrimonia, White Flower Patrinia
Herb etc., consist of glycoside, saponin and flavone and could inhibit proliferation on HepG2, A549, 4T1 and CT26 cell lines, and decrease $\mathrm{p}-\mathrm{AKT}$ and $\mathrm{mTOR}$ protein expression.

In this report, the late-stage patients received acceptable treatment result by using herbal extractive medicines as second or later treatment, which provide moderate effective and low-toxicity, and it could be considered as an as complementary and alternative medicine. In PC group, the median and the average survival time were 5.1 and 6.5 months; The 3-, 6-, and 10-month survival rates were $92.3 \%, 46.2 \%$, and $30.8 \%$, respectively. The longest survival time was 16.7 months and patient still alive. In HCC group, the average and median survival time were 12.5 and 5.0 months; The 3 -, 6-, and 12-month survival time rates were $77.4 \%, 38.7 \%$, and $29.0 \%$, respectively. The longest survival time was 84.2 months. In LC group, the 6 months, 1-, 2- and 5-year survival time rates were $33.9 \%, 19.1 \%, 14.3 \%$ and $4.2 \%$, respectively. Meanwhile, in lung cancer group, once the survival time was over 6 months, the average and median survival time were 29.98 and 14.8 months; and the 1-, 2- and 5-year survival time rates were up to $56.1 \%, 42.1 \%$ and $12.3 \%$, respectively. Meanwhile, the most common drugrelated adverse event was gastrointestinal, especially dyspepsia, and just a low percentage of patients reported fatigue or diarrhea. No hematology side-effect, which was up to grade 2, was observed.

In summary, early involvement of palliative care can lead to less utilization of useless care caused by severe side-effects. Not only, in 2012, the American Society for Clinical Oncology (ASCO) has made a recommendation for 'combined standard oncology care and palliative care consideration early in the course of illness for any patient with metastatic cancer and/or high symptom burden [24, 25], but also Chinese herbal medicine(CHM) may be considered as an add-on to conventional medicine in the management of pain, constipation, anorexia and fatigue in cancer patients. Thus, early palliative care was not ought to be recognized as a final method, and it was equally important to chemotherapy. This study makes an important contribution to the body of evidence on the efficacy of herbal extractive medicine that acted as an alternative method in late-stage lung, pancreatic and liver cancer which could be recommended as an initial treatment as second line or later treatment. Future RCTs should improve outcome measurement and report detailed safety outcomes.

\section{Conclusion}

CHM can be considered as complementary and alternative medicine that provide moderate effective and low toxicity for advanced cancer.

\section{Acknowledgment}

None.

\section{Conflict of Interest}

The authors declare that there is no conflict of interest.

\section{References}

1. Lin QJ, Feng Y, Jin C, Fu DL (2015) Current status and progress of pancreatic cancer in China. World J Gastroenterol 21(26): 7988-8003. 
2. Bruix J, Gores GJ, Mazzaferro V (2014) Hepatocellular carcinoma: clinical frontiers and perspectives. Gut 63(5): 844-855.

3. Poon RT, Fan ST, Lo CM, Liu CL, Wong J (2002) Long-term survival and pattern of recurrence after resection of small hepatocellular carcinoma in patients with preserved liver function: implications for a strategy of salvage transplantation. Ann Surg 235(3): 373-382.

4. Hung H (2005) Treatment modalities for hepatocellular carcinoma. Curr Cancer Drug Targets 5(2): 131-138.

5. Sheng Long Ye, Yang J, Bie P, Zhang S, Chen X, et al. (2018) Safety assessment of sorafenib in Chinese patients with unresectable hepatocellular carcinoma: subgroup analysis of the GIDEON study. BMC Cancer 2018, 18(1): 247-258.

6. Baxter NN, Whitson BA, Tuttle TM (2007) Trends in the treatment and outcome of pancreatic cancer in the United States. Ann Surg Oncol 14(4): 1320-1326.

7. Kanai M, Yoshimura K, Asada M, Imaizumi A, Suzuki C, et al. (2011) A phase I/II study of gemcitabine-based chemotherapy plus curcumin for patients with gemcitabine-resistant pancreatic cancer. Cancer Chemother Pharmacol 68(1): 157-164.

8. Garassino MC, Cho BC, Kim JH, Mazières J, Vansteenkiste J, et al. (2018) Durvalumab as third-line or later treatment for advanced non-smallcell lung cancer (ATLANTIC): an open-label, single-arm, phase 2 study. Lancet Oncol 19(4): 521-536.

9. Pu WL, Sun LK, Gao XM, Rüegg C, Cuendet M, et al. (2017) Targeting tumor-associated macrophages by anti-tumor Chinese materia medica. Chin J Integr Med 23(10): 723-732.

10. Du J, Chen BC, Fu XQ, Su T, Li T, et al. (2016) In vitro assays suggest Shenqi Fuzheng Injection has the potential to alter melanoma immune microenvironment. J Ethnopharmacol 194: 15-19.

11. Li W, Chen C, Saud SM, Geng L, Zhang G, et al. (2014) Fei-Liu-Ping ointment inhibits lung cancer growth and invasion by suppressing tumor inflammatory microenvironment. BMC Complementary and Alternative Medicine 14(1):153.

12. Leighl NB (2012) Treatment paradigms for patients with metastatic non-small-cell lung cancer: first-, second-, and third-line. Curr Oncol 19(Suppl 1): S52-58.
13. Torgerson S, Wiebe LA (2013) Supportive care of the patient with advanced pancreatic cancer. Oncology (Williston Park) 27(3): 183-190.

14. Palliative care: Overview of fatigue, weakness, and asthenia

15. Chung VC, Wu X, Lu P, Hui EP, Zhang Y, et al. (2016) Chinese Herbal Medicine for Symptom Management in Cancer Palliative Care: Systematic Review and Meta-analysis. Medicine 95(7): e2793.

16. Bakitas M, Lyons KD, Hegel MT, Balan S, Brokaw FC, et al (2009) Effects of a palliative care intervention on clinical outcomes in patients with advanced cancer: The Project ENABLE II randomized controlled trial. JAMA 302(7): 741-749.

17. Temel JS, Greer JA, Muzikansky A, Gallagher ER, Admane S, et al. (2010) Early palliative care for patients with metastatic non-small-cell lung cancer. N Engl J Med 363(8): 733-742.

18. Maltoni M, Scarpi E, Dall’Agata M, Zagonel V, Bertè R, et al. (2016) Systematic versus on-demand early palliative care: results from a multicentre, randomised clinical trial. Eur J Cancer 65: 61-68.

19. Bakitas MA, Tosteson TD, Li Z, Lyons KD, Hull JG, et al. (2015) Early versus delayed initiation of concurrent palliative oncology care: patient outcomes in the ENABLE III randomized controlled trial. J Clin Oncol 33(13): 1438-1445.

20. Zimmermann C, Swami N, Krzyzanowska M, Hannon B, Leighl N, et al (2014) Early palliative care for patients with advanced cancer: a clusterrandomised controlled trial. Lancet 383(9930): 1721-1730.

21. Fulton J, LeBlanc T, Porter Starr KN, Kamal A, Ramos K, et al. (2017) Integrated Outpatient Palliative Care in Oncology.

22. Erdek MA, King LM, Ellsworth SG (2013) Pain management and palliative care in pancreatic cancer. Current Problems in Cancer, 37(5): 266-272.

23. Rabow MW, Petzel MQB, Adkins SH (2017) Symptom Management and Palliative Care in Pancreatic Cancer. Cancer Journal 23(6): 362.

24. Bruera E, Yennurajalingam S (2012) Palliative care in advanced cancer patients: how and when? Oncologist 17(2): 267-273.

25. Smith T, Temin S, Alesi ER, Abernethy AP, Balboni (2012) TA American Society of Clinical Oncology provisional clinical opinion: the integration of palliative care into standard oncology care. J Clin Oncol 30(8): 880887. 